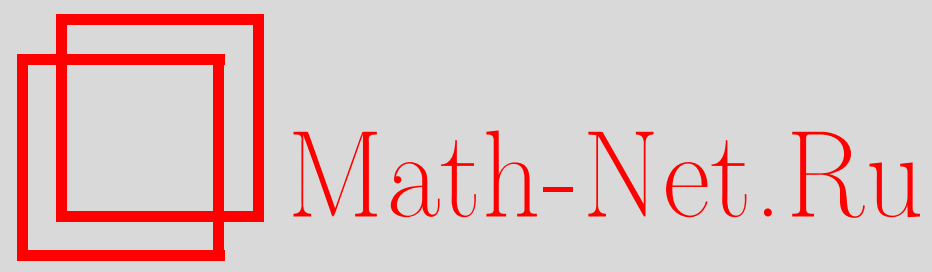

А. М. Зубков, Оценки расстояния по вариации между распределениями двух наборов независимых случайных величин, Матем. вопр. криптогр., 2020, том 11, выпуск 3, 21-29

DOI: https://doi.org/10.4213/mvk329

Использование Общероссийского математического портала Math-Net.Ru подразумевает, что вы прочитали и согласны с пользовательским соглашением http://www.mathnet.ru/rus/agreement

Параметры загрузки:

IP: 52.6 .47 .48

26 апреля 2023 г., $12: 33: 27$

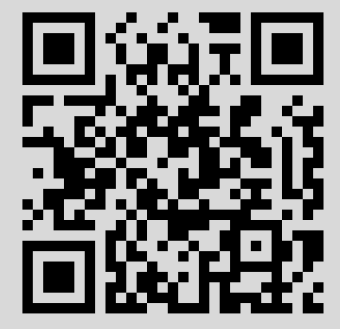


МАТЕМАТИЧЕСКИЕ ВОПРОСЫ КРИПТОГРАФИИ

2020 T. 11 № 3 C. 21-29

УДК 519.213.21

DOI https://doi.org/10.4213/mvk329

\title{
Оценки расстояния по вариации между распределениями двух наборов независимых случайных величин
}

\author{
А. М. Зубков
}

Математический институт им.В.А.Стеклова РАН, Москва

Получено 29.IV.2019

Аннотация. Получены явные нижние и верхние оценки расстояний по вариации между распределениями наборов $\left(X_{1}, \ldots, X_{n}\right)$ и $\left(Y_{1}, \ldots, Y_{n}\right)$ независимых, но не обязательно одинаково распределенных (внутри каждого набора) случайных величин в терминах расстояний по вариации $\rho_{k}$ между распределениями отдельных компонент $X_{k}$ и $Y_{k}, k=1, \ldots, n$. Оценки для случая однородных выборок были опубликованы в этом журнале в 2018 г. Качественно полученные в настоящей работе оценки согласуются с оценками для однородных случаев.

Ключевые слова: расстояние по вариации, неоднородные выборки, вероятностные неравенства, двусторонние оценки

Estimates for the variational distance between two sets of independent random variables

\section{A. M. Zubkov}

Steklov Mathematical Institute of RAS, Moscow

Abstract. We obtain explicit lower and upper estimates of the total variation distance between distributions of two sets $\left(X_{1}, \ldots, X_{n}\right)$ and $\left(Y_{1}, \ldots, Y_{n}\right)$ of independent random variables which may be nonidentically distributed inside each set. Estimates are formulated in terms of total variation distances $\rho_{k}$ between distributions of separate components $X_{k}$ and $Y_{k}, k=1, \ldots, n$. Results for the case of homogeneous samples was considered in this journal in 2018. On the qualitative level the estimates of the present paper correspond with the estimates obtained for the homogeneous cases earlier.

Keywords: total variation distance, nonhomogeneous samples, probabilistic inequalities, two-side estimates 


\section{1. Введение}

Расстояние по вариации является естественной мерой возможности различения простых статистических гипотез. Обычно предполагается, что наблюдения независимы и одинаково распределены. Однако при применении к независимым выборкам пакетов статистических критериев статистики этих критериев оказываются независимыми, но не одинаково распределенными. Поэтому оценки расстояния по вариации между наборами независимых, но не обязательно одинаково (внутри набора) распределенных случайных величин представляют интерес как с теоретической, так и с прикладной точки зрения.

В [7] были получены верхние и нижние оценки расстояния по вариации между распределениями наборов независимых одинаково (в каждом наборе) распределенных случайных величин в терминах расстояния по вариации $\rho$ между отдельными элементами этих наборов и чисел элементов в этих наборах. При довольно широких условиях как верхние, так и нижние оценки расстояния по вариации между распределениями этих наборов с ростом числа $n$ элементов в них увеличивались как $\rho \sqrt{n}$ (пока оставались меньше 1). Это согласуется с оценками необходимого объема выборки для ряда конкретных критериев.

В настоящей работе получены нижние и верхние оценки расстояний по вариации между распределениями наборов $\left(X_{1}, \ldots, X_{n}\right)$ и $\left(Y_{1}, \ldots, Y_{n}\right)$ независимых, но не обязательно одинаково распределенных случайных величин в терминах расстояний по вариации $\rho_{k}$ между распределениями отдельных компонент $X_{k}$ и $Y_{k}, k=1, \ldots, n$. Изза неоднородности распределений случайных величин в наборах изменяются формы и степень точности оценок, а также способы доказательств. Качественно полученные в этой работе оценки согласуются с оценками из [7], если элементы наборов одинаково распределены.

\section{2. Формулировки результатов}

Для случайных величин $X$ и $Y$, принимающих значения в одном и том же пространстве $B$ с $\sigma$-алгеброй $\mathcal{B}$ измеримых множеств, расстояние по вариации между их распределениями $P_{X}$ и $P_{Y}$ определяется равенством

$$
\rho(X, Y)=\rho\left(P_{X}, P_{Y}\right)=\sup _{A \in \mathcal{B}}\left|P_{X}(A)-P_{Y}(A)\right| .
$$


Хорошо известно (см., например, $[1,5,6])$, что если $X$ и $Y$ - случайные величины с не более чем счетным множеством значений $B$, то

$$
\rho(X, Y)=\frac{1}{2} \sum_{b \in B}|\mathbf{P}\{X=b\}-\mathbf{P}\{Y=b\}|,
$$

поэтому если $C=C\left(P_{X}, P_{Y}\right)=\{b \in B: \mathbf{P}\{X=b\}>\mathbf{P}\{Y=b\}\}$, то

$$
\rho(X, Y)=P_{X}(C)-P_{Y}(C)=P_{Y}(B \backslash C)-P_{X}(B \backslash C),
$$

кроме того, если $X_{1}, \ldots, X_{m}$ и $Y_{1}, \ldots, Y_{m}$ - два набора независимых случайных величин, принимающих значения в одном и том же пространстве $B$, а $g\left(x_{1}, \ldots, x_{m}\right)$ - измеримая функция, определенная на $B^{m}$, TO

$$
\begin{gathered}
\rho\left(g\left(X_{1}, \ldots, X_{m}\right), g\left(Y_{1}, \ldots, Y_{m}\right)\right) \leqslant \rho\left(\left(X_{1}, \ldots, X_{m}\right),\left(Y_{1}, \ldots, Y_{m}\right)\right) \\
\leqslant \rho\left(X_{1}, Y_{1}\right)+\ldots+\rho\left(X_{m}, Y_{m}\right) .
\end{gathered}
$$

Для вероятностных мер $P_{X}$ и $P_{Y}$ на $B$ и соответствующего им множества $C=C\left(P_{X}, P_{Y}\right)$ из (3) введем функцию

$$
v=v\left(P_{X}, P_{Y}\right)=\min \left\{P_{X}(C), P_{Y}(C), P_{X}(\Omega \backslash C), P_{Y}(\Omega \backslash C)\right\},
$$

тогда $v \leqslant \frac{1}{2}\left(1-\rho\left(P_{X}, P_{Y}\right)\right)$, так как если, например, $v=P_{X}(C)>$ $\frac{1}{2}\left(1-\rho\left(P_{X}, P_{Y}\right)\right)$, то

$P_{Y}(C)=v+\rho\left(P_{X}, P_{Y}\right), \quad P_{Y}(\Omega \backslash C)=1-v-\rho\left(P_{X}, P_{Y}\right)<\frac{1}{2}\left(1-\rho\left(P_{X}, P_{Y}\right)\right)$.

В [7] были доказаны две теоремы, усиливающие аналогичные утверждения из препринтов [3] и [4].

Теорема 1 ([7]). Пусть $X_{1}, X_{2}, \ldots$ - независимые целочисленные случайные величины с одним и тем же распределением $P$ и $Y_{1}, Y_{2}, \ldots$ независимые иелочисленные случайные величины с одним и тем же распределением $Q, \rho(P, Q)=\varepsilon>0$. Справедливы неравенства

$$
\begin{aligned}
& \rho\left(P^{n}, Q^{n}\right)=\rho\left(\left(X_{1}, \ldots, X_{n}\right),\left(Y_{1}, \ldots, Y_{n}\right)\right) \\
& \quad \geqslant \frac{e^{-4 / n v(1-v)}}{2(1+2 / n)} \sqrt{\frac{v}{1-v}}\left(\Phi(2 \varepsilon \sqrt{n})-\frac{1}{2}\right),
\end{aligned}
$$

где $v=v(P, Q), 2 \leqslant n v<\frac{n-1}{2} u \Phi(x)=\frac{1}{\sqrt{2 \pi}} \int_{-\infty}^{x} e^{-u^{2} / 2} d u-$ бункция стандартного нормального распределения. 
Замечание 1. Разность $\Phi(2 \varepsilon \sqrt{n})-\frac{1}{2}$ не превышает $\frac{1}{2}$ и эквивалентна $\varepsilon \sqrt{2 n / \pi}$, если $\varepsilon \sqrt{n} \rightarrow 0$, т. е. нижняя оценка при $n \rightarrow \infty$ в области своих малых значений растет по порядку как $\sqrt{n}$.

Теорема 2 ([7]). Пусть $X_{1}, X_{2}, \ldots u Y_{1}, Y_{2}, \ldots$ - независимые случайные величины, принимающие значения $1, \ldots, N$ :

$$
\mathbf{P}\left\{X_{t}=k\right\}=p_{k}, \quad \mathbf{P}\left\{Y_{t}=k\right\}=r_{k}, \quad k \in\{1, \ldots, N\}, \quad t=1,2 \ldots,
$$

u $\rho=\rho\left(X_{1}, Y_{1}\right)=\frac{1}{2} \sum_{k=1}^{N}\left|p_{k}-r_{k}\right|$. Тогда

$$
\rho\left(\left(X_{1}, \ldots, X_{n}\right),\left(Y_{1}, \ldots, Y_{n}\right)\right) \leqslant \rho \sqrt{n}\left(\frac{1}{\sqrt{P}+\sqrt{P+\rho}}+\frac{1}{\sqrt{R}+\sqrt{R+\rho}}\right),
$$

где $P=\sum_{k=1}^{N} p_{k} \mathbb{I}\left\{p_{k}<r_{k}\right\}, R=\sum_{k=1}^{N} r_{k} \mathbb{I}\left\{p_{k}>r_{k}\right\}$.

Замечание 2. Так как

$$
\begin{gathered}
P+R=\sum_{k=1}^{n} \min \left\{p_{k}, r_{k}\right\}=1-\sum_{k=1}^{n} \max \left\{p_{k}, r_{k}\right\}, \\
\sum_{k=1}^{n} \max \left\{p_{k}, r_{k}\right\}-\sum_{k=1}^{n} \min \left\{p_{k}, r_{k}\right\}=2 \rho,
\end{gathered}
$$

то $P+R=\sum_{k=1}^{n} \min \left\{p_{k}, r_{k}\right\}=\frac{1}{2}(1-\rho)$. Константа в правой части тем больше, чем меньше $\min \{P, R\}$.

В настоящей работе получены нижние и верхние оценки для расстояний по вариации между наборами независимых случайных величин, возможно, имеющих разные распределения.

Теорема 3. Пусть $X_{1}, X_{2}, \ldots, X_{n}$ u $Y_{1}, Y_{2}, \ldots, Y_{n}-$ два набора независимых случайных величин, принимающих значения $1, \ldots, N$ :

$\mathbf{P}\left\{X_{k}=j\right\}=p_{j}^{(k)}, \quad \mathbf{P}\left\{Y_{k}=j\right\}=r_{j}^{(k)}, \quad j \in\{1, \ldots, N\}, \quad k=1,2 \ldots, n$, $u \rho_{k}=\rho\left(X_{k}, Y_{k}\right)=\frac{1}{2} \sum_{j=1}^{N}\left|p_{j}^{(k)}-r_{j}^{(k)}\right|>0(k=1, \ldots, n), S=\sum_{k=1}^{n} \rho_{k}$. Тогда

$$
\rho\left(\left(X_{1}, \ldots, X_{n}\right),\left(Y_{1}, \ldots, Y_{n}\right)\right) \geqslant 1-2 \exp \left\{-\frac{S^{2}}{2 n}\right\} .
$$

Замечание 3 . Если $\rho_{1}=\ldots=\rho_{n}=\rho<1$, то оценка принимает вид

$$
\rho\left(\left(X_{1}, \ldots, X_{n}\right),\left(Y_{1}, \ldots, Y_{n}\right)\right) \geqslant 1-2 \exp \left\{-\frac{1}{2} n \rho^{2}\right\}
$$


правая часть стремится к 1 при $n \rightarrow \infty$, но отрицательна при $n<\frac{2}{\rho^{2}} \ln 2$. Доказательство теоремы 3 основано на более грубых оценках, чем в [7], где существенно использовались особенности биномиального распределения. Неравенство (6) позволяет оценивать объем выборки, достаточный для различения гипотез.

Теорема 4. Пусть $X_{1}, X_{2}, \ldots, X_{n} u Y_{1}, Y_{2}, \ldots, Y_{n}$ - независимье случайные величины, принимающие значения $1, \ldots, N$ :

$$
\begin{gathered}
\mathbf{P}\left\{X_{t}=k\right\}=p_{k}^{(t)}, \quad \mathbf{P}\left\{Y_{t}=k\right\}=r_{k}^{(t)}, \quad k \in\{1, \ldots, N\}, \quad t=1,2 \ldots, n, \\
\rho_{t}=\rho\left(X_{t}, Y_{t}\right)=\frac{1}{2} \sum_{k=1}^{N}\left|p_{k}^{(t)}-r_{k}^{(t)}\right| t=1, \ldots, n, u \\
\min _{1 \leqslant t \leqslant n} \min \left\{P_{t}, R_{t}\right\} \geqslant \delta>0, \quad P_{t}=\sum_{k: p_{k}^{(t)}<r_{k}^{(t)}} p_{k}^{(t)}, R_{t}=\sum_{k: r_{k}^{(t)}<p_{k}^{(t)}} r_{k}^{(t)} .
\end{gathered}
$$

Tогда

$$
\rho\left(\left(X_{1}, \ldots, X_{n}\right),\left(Y_{1}, \ldots, Y_{n}\right)\right) \leqslant \frac{1}{\sqrt{2 \delta}} \sqrt{\sum_{t=1}^{n} \rho_{t}^{2}} .
$$

Замечание 4. Довольно жесткое условие $\min _{1 \leqslant t \leqslant n} \min \left\{P_{t}, R_{t}\right\} \geqslant \delta>0$, из-за которого увеличивается коэффициент в верхней оценке теоремы 4, введено с учетом замечания 2 и для упрощения вида этой оценки. Теорема 4 позволяет оценивать объем выборки, необходимый для различения двух простых гипотез.

\section{3. Доказательства теорем}

Доказательство теоремы 3. Для каждого $k=1, \ldots, n$ выберем множество $C_{k} \subset\{1, \ldots, N\}$ в соответствии с (3) так, чтобы выполнялись условия

$$
\mathbf{P}\left\{X_{k} \in C_{k}\right\}-\mathbf{P}\left\{Y_{k} \in C_{k}\right\}=\rho_{k}, \quad k=1, \ldots, n .
$$

При $\mathbf{x}=\left(x_{1}, \ldots, x_{n}\right)$ рассмотрим функцию

$$
f_{n}(\mathbf{x}) \stackrel{\text { def }}{=} f\left(x_{1}, \ldots, x_{n}\right)=\sum_{k=1}^{n} \mathbb{I}\left\{x_{k} \in C_{k}\right\}
$$

Тогда при $\mathbf{X}=\left(X_{1}, \ldots, X_{n}\right), \mathbf{Y}=\left(Y_{1}, \ldots, Y_{n}\right)$ случайные величины $f_{n}(\mathbf{X})$ и $f_{n}(\mathbf{Y})$ как суммы $n$ индикаторов каждая принимают значения $0,1, \ldots, n$, причем согласно (4) и определению (1)

$$
\rho(\mathbf{X}, \mathbf{Y}) \geqslant \rho\left(f_{n}(\mathbf{X}), f_{n}(\mathbf{Y})\right) \geqslant \max _{0 \leqslant x \leqslant n}\left|\mathbf{P}\left\{f_{n}(\mathbf{X}) \geqslant x\right\}-\mathbf{P}\left\{f_{n}(\mathbf{Y}) \geqslant x\right\}\right| .
$$


Чтобы оценить правую часть (8), используем следующие соображения. В силу (7) $\mathbf{P}\left\{X_{k} \in C_{k}\right\}-\mathbf{P}\left\{Y_{k} \in C_{k}\right\}=\rho_{k} \geqslant 0, k=1, \ldots, n$, и

$$
\mathbf{M} f_{n}(\mathbf{X})=\mathbf{M} f_{n}(\mathbf{Y})+S, \quad S=\sum_{k=1}^{n} \rho_{k} .
$$

Положим в (8)

$$
u=\frac{\mathbf{M} f_{n}(\mathbf{X})+\mathbf{M} f_{n}(\mathbf{Y})}{2}=\mathbf{M} f_{n}(\mathbf{Y})+\frac{1}{2} S=\mathbf{M} f_{n}(\mathbf{X})-\frac{1}{2} S .
$$

Из неравенств Хефдинга [2]: если $S_{n}=\xi_{1}+\ldots+\xi_{n}$, где $\xi_{1}, \ldots, \xi_{n}$ независимы и $\mathbf{P}\left\{0 \leqslant \xi_{k} \leqslant 1\right\}=1$ для всех $k=1, \ldots, n$, то

$$
\begin{gathered}
\mathbf{P}\left\{S_{n}-\mathbf{M} S_{n} \geqslant z\right\} \leqslant e^{-2 z^{2} / n}, \quad z \in\left[0, n-\mathbf{M} S_{n}\right), \\
\mathbf{P}\left\{S_{n}-\mathbf{M} S_{n} \leqslant-z\right\} \leqslant e^{-2 z^{2} / n}, \quad z \in\left[0, \mathbf{M} S_{n}\right),
\end{gathered}
$$

получаем оценки

$$
\begin{aligned}
& \mathbf{P}\left\{f_{n}(\mathbf{X}) \leqslant u\right\}=\mathbf{P}\left\{f_{n}(\mathbf{X})-\mathbf{M} f_{n}(\mathbf{X}) \leqslant-\frac{1}{2} S\right\} \leqslant \exp \left\{-\frac{1}{2 n} S^{2}\right\} \\
& \mathbf{P}\left\{f_{n}(\mathbf{Y}) \leqslant u\right\}=1-\mathbf{P}\left\{f_{n}(\mathbf{Y})-\mathbf{M} f_{n}(\mathbf{Y}) \geqslant \frac{1}{2} S\right\} \geqslant 1-\exp \left\{-\frac{1}{2 n} S^{2}\right\} .
\end{aligned}
$$

Из них следует, что

$$
\mathbf{P}\left\{f_{n}(\mathbf{X}) \geqslant x\right\}-\mathbf{P}\left\{f_{n}(\mathbf{Y}) \geqslant x\right\} \geqslant 1-2 \exp \left\{-\frac{1}{2 n} S^{2}\right\} .
$$

Отсюда и из (8) следует утверждение теоремы 3.

Доказательство теоремы 4. Доказательство проводится по той же схеме, что и в [7]. Используя определение расстояния по вариации и то, что функция $\prod_{t=1}^{n}\left(r_{k_{t}}^{(t)}+\left(p_{k_{t}}^{(t)}-r_{k_{t}}^{(t)}\right) u\right)$ при изменении $u$ от 0 до 1 изменяется от $\prod_{j=1}^{n} r_{k_{j}}^{(t)}$ до $\prod_{t=1}^{n} p_{k_{j}}^{(t)}$, получаем:

$$
\begin{gathered}
\rho\left(\left(X_{1}, \ldots, X_{n}\right),\left(Y_{1}, \ldots, Y_{n}\right)\right)=\frac{1}{2} \sum_{k_{1}, \ldots, k_{n}=1}^{N}\left|\prod_{t=1}^{n} p_{k_{j}}^{(t)}-\prod_{j=1}^{n} r_{k_{j}}^{(t)}\right| \\
=\frac{1}{2} \sum_{k_{1}, \ldots, k_{n}=1}^{N}\left|\int_{0}^{1} \frac{d}{d u} \prod_{t=1}^{n}\left(r_{k_{t}}^{(t)}+\left(p_{k_{t}}^{(t)}-r_{k_{t}}^{(t)}\right) u\right) d u\right| .
\end{gathered}
$$


Далее, при $\theta_{k}^{(t)}=p_{k}^{(t)}-r_{k}^{(t)}$

$$
\frac{d}{d u} \prod_{t=1}^{n}\left(r_{k_{t}}^{(t)}+\theta_{k_{t}}^{(t)} u\right)=\sum_{m=1}^{n} \frac{\theta_{k_{m}}^{(m)}}{r_{k_{m}}^{(m)}+\theta_{k_{m}}^{(m)} u} \prod_{t=1}^{n}\left(r_{k_{t}}^{(t)}+\theta_{k_{t}}^{(t)} u\right)
$$

Поэтому

$$
\begin{aligned}
\mid \prod_{t=1}^{n} p_{k_{t}}^{(t)} & -\prod_{t=1}^{n} r_{k_{t}}^{(t)}|=| \int_{0}^{1} \sum_{m=1}^{n} \frac{\theta_{k_{m}}^{(m)}}{r_{k_{m}}^{(m)}+\theta_{k_{m}}^{(m)} u} \prod_{t=1}^{n}\left(r_{k_{t}}^{(t)}+\theta_{k_{t}}^{(t)} u\right) d u \mid \\
& \leqslant \int_{0}^{1}\left|\sum_{m=1}^{n} \frac{\theta_{k_{m}}^{(m)}}{r_{k_{m}}^{(m)}+\theta_{k_{m}}^{(m)} u}\right| \prod_{t=1}^{n}\left(r_{k_{t}}^{(t)}+\theta_{k_{t}}^{(t)} u\right) d u .
\end{aligned}
$$

Значит,

$$
\begin{gathered}
\rho\left(\left(X_{1}, \ldots, X_{n}\right),\left(Y_{1}, \ldots, Y_{n}\right)\right) \\
\leqslant \frac{1}{2} \int_{0}^{1} \sum_{k_{1}, \ldots, k_{n}=1}^{N}\left|\sum_{m=1}^{n} \frac{\theta_{k_{m}}^{(m)}}{r_{k_{m}}^{(m)}+\theta_{k_{m}}^{(m)} u}\right| \prod_{t=1}^{n}\left(r_{k_{t}}^{(t)}+\theta_{k_{t}}^{(t)} u\right) d u .
\end{gathered}
$$

Пусть $Z_{1}(u), \ldots, Z_{n}(u)$ - независимые случайные величины со значениями в множестве $\{1, \ldots, N\}$ и распределениями

$$
\mathbf{P}\left\{Z_{m}(u)=k\right\}=r_{k}^{(m)}+\theta_{k}^{(m)} u, \quad k \in\{1, \ldots, N\}, m=1, \ldots, n .
$$

Тогда выражение под знаком интеграла в (11) представляет собой математическое ожидание случайной величины $\left|\sum_{m=1}^{n} \frac{\theta_{Z_{m}(u)}^{(m)}}{r_{Z_{m}(u)}^{(m)}+\theta_{Z_{m}(u)}^{(m)} u}\right|$, т. е.

$$
\rho\left(\left(X_{1}, \ldots, X_{n}\right),\left(Y_{1}, \ldots, Y_{n}\right)\right) \leqslant \frac{1}{2} \int_{0}^{1} \mathbf{M}\left|\sum_{m=1}^{n} \frac{\theta_{Z_{m}(u)}^{(m)}}{r_{Z_{m}(u)}^{(m)}+\theta_{Z_{m}(u)}^{(m)} u}\right| d u
$$

Так как по определению $\theta_{k}^{(m)}=p_{k}^{(m)}-r_{k}^{(m)}, k \in\{1, \ldots, N\}$, $m=1, \ldots, n$, то

$\mathbf{M} \frac{\theta_{Z_{m}(u)}^{(m)}}{r_{Z_{m}(u)}^{(m)}+\theta_{Z_{m}(u)}^{(m)} u}=\sum_{k=1}^{N} \mathbf{P}\left\{Z_{m}(u)=k\right\} \frac{\theta_{k}^{(m)}}{r_{k}^{(m)}+\theta_{k}^{(m)} u}=\sum_{k=1}^{N}\left(p_{k}^{(m)}-r_{k}^{(m)}\right)=0$ 


$$
\begin{gathered}
\mathbf{M}\left(\frac{\theta_{Z_{m}(u)}^{(m)}}{r_{Z_{m}(u)}^{(m)}+\theta_{Z_{m}(u)}^{(m)} u}\right)^{2} \\
\quad=\sum_{k=1}^{N} \mathbf{P}\left\{Z_{m}(u)=k\right\}\left(\frac{\theta_{k}^{(m)}}{r_{k}^{(m)}+\theta_{k}^{(m)} u}\right)^{2}=\sum_{k=1}^{N} \frac{\left(\theta_{k}^{(m)}\right)^{2}}{r_{k}^{(m)}+\theta_{k}^{(m)} u},
\end{gathered}
$$

отсюда и из независимости случайных величин $Z_{1}(u), \ldots, Z_{n}(u)$ следует, что

$$
\begin{aligned}
& \mathbf{M}\left|\sum_{m=1}^{n} \frac{\theta_{Z_{m}(u)}^{(m)}}{r_{Z_{m}(u)}^{(m)}+\theta_{Z_{m}(u)}^{(m)} u}\right| \leqslant \sqrt{\mathbf{M}\left(\sum_{m=1}^{n} \frac{\theta_{Z_{m}(u)}^{(m)} \frac{\theta_{Z_{m}(u)}^{(m)}+\theta_{Z_{m}(u)}^{(m)} u}{r^{(m)}}}{2}\right.} \\
& =\sqrt{\sum_{m=1}^{n} \mathbf{M}\left(\frac{\theta_{Z_{m}(u)}^{(m)}}{r_{Z_{m}(u)}^{(m)}+\theta_{Z_{m}(u)}^{(m)} u}\right)^{2}}=\sqrt{\sum_{m=1}^{n} \sum_{k=1}^{N} \frac{\left(\theta_{k}^{(m)}\right)^{2}}{r_{k}^{(m)}+\theta_{k}^{(m)} u} .}
\end{aligned}
$$

В [7] было показано, что если $\rho_{m}=\frac{1}{2} \sum_{k=1}^{N}\left|p_{k}^{(m)}-r_{k}^{(m)}\right|$ и

$$
P_{m}=\sum_{\substack{k \in\{1, \ldots, N\} \\ p_{k}^{(m)}>r_{k}^{(m)}}} p_{k}^{(m)}, \quad R_{m}=\sum_{\substack{k \in\{1, \ldots, N\} \\ p_{k}^{(m)}<r_{k}^{(m)}}} r_{k}^{(m)}
$$

TO

$$
\sum_{k=1}^{N} \frac{\left(\theta_{k}^{(m)}\right)^{2}}{r_{k}^{(m)}+\theta_{k}^{(m)} u} \leqslant\left(\frac{1}{P_{m}+u \rho_{m}}+\frac{1}{R_{m}+u \rho_{m}}\right) \rho_{m}^{2}<\frac{2 \rho_{m}^{2}}{\min \left\{P_{m}, R_{m}\right\}} .
$$

Подставляя эти оценки в (13) и учитывая условие $\min _{1 \leqslant m \leqslant n} \min \left\{P_{m}, R_{m}\right\} \geqslant \delta$, получаем при всех $u \in[0,1]$ :

$$
\mathbf{M}\left|\sum_{m=1}^{n} \frac{\theta_{Z_{m}(u)}^{(m)}}{r_{Z_{m}(u)}^{(m)}+\theta_{Z_{m}(u)}^{(m)} u}\right| \leqslant \sqrt{\frac{2}{\delta} \sum_{m=1}^{n} \rho_{m}^{2}} .
$$

Из последней оценки и из (12) следует, что $\rho\left(\left(X_{1}, \ldots, X_{n}\right),\left(Y_{1}, \ldots, Y_{n}\right)\right) \leqslant \frac{1}{2} \int_{0}^{1} \sqrt{\frac{2}{\delta} \sum_{m=1}^{n} \rho_{m}^{2}} d u=\frac{1}{\sqrt{2 \delta}} \sqrt{\sum_{m=1}^{n} \rho_{m}^{2}}$. Теорема 4 доказана. 


\section{Список литературы}

[1] Золотарев В.М., Современная теория суммирования независимых случайных величин, M. : Наука, 1986, 416 с.

[2] Hoeffding W., "Probability inequalities for sums of bounded random variables", J. Amer. Statist. Assoc., 58:301 (1963), 13-30.

[3] Reyzin L., A note on the statistical difference of small direct products, Boston Univ. Computer Science, Techn. Rep. BUCS-TR-2004-032.

[4] Renner R., On the variational distance of independently repeated experiments, arXiv: cs.IT/0509013.

[5] Sahai A., Vadhan S., "Manipulating statistical difference", Randomization Methods in Algorithm Design, DIMACS Workshop, December 1997, DIMACS Ser. in Discr. Math. and Theor. Comput. Sci., 43, Amer. Math. Soc., Providence, R.I., 1999, 251-270.

[6] Sahai A., Vadhan S., "A complete problem for statistical zero knowledge", J. ACM, 50:2 (2003), 196-249.

[7] Зубков А. М., "Новые оценки расстояния по вариации между двумя распределениями выборки", Математические вопросы криптографии, 9:3 (2018), 45-60. 
